\title{
Optimizing systematic technology adoption with heterogeneous agents
}

\author{
Huayi Chen ${ }^{1}$, Tieju Ma ${ }^{1,2, *}$ \\ ${ }^{1}$ East China University of Science and Technology, \\ Meilong Road 130, Shanghai 200237, China \\ ${ }^{2}$ International Institute for Applied System Analysis \\ Schlosplatz 1 A-2361 Laxenburg, Austria
}

\begin{abstract}
The traditional operational optimization models of systematic technology adoption commonly assume the existence of a global social planner and ignore the existence of heterogeneous decision makers who interact with each other. This paper develops a stylized (or conceptual) optimization model of systematic technology adoption with heterogeneous agents (i.e., decision makers) and uncertain technological learning. Each agent attempts to identify optimal solutions to adopting technologies for a portion of the entire system. The agents in the model have different foresight and different risk attitudes and interact with one another in terms of technological spillover. This paper first illustrates that although a well recalibrated representative model can perform well enough when the interest is placed on aggregate variables, it could react to a policy (a carbon tax in this paper) differently from the multi-agent model. Then this paper explores how the agents' heterogeneities and interactions affect the optimal solutions of systematic technology adoption. The main findings of the study are that (1) the existence of multiple agents implies a slower adoption of advanced technologies in the entire system than assuming the existence of a global social planner, (2) with homogeneous agents, technological spillover tends to enhance the lock-in effect on previous technologies, and (3) with heterogeneous agents, even a small technological spillover rate can significantly accelerate the adoption of the advanced technology.
\end{abstract}

Keywords: OR in societal problem analysis, systematic technology adoption, optimization, heterogeneous agents, uncertain technological learning

\section{Introduction}

The adoption of new technologies has long been believed and shown to be one of the most important sources of economic growth, long-run productivity and sustainable development (e.g., Metcalfe 1987, Freeman 1994, Mokyr 1990, World Bank, 2000). Studies on technology adoption can be grouped into one of two streams. The first stream addresses the psychology-based acceptance of

\footnotetext{
* Corresponding author at: School of Business, East China University of Science and Technology, Meilong Road 130, Shanghai 200237, China. Tel.: +86 2164252015

Email: tjma@ecust.edu.cn
} 
new technologies by individual users or organizations. The well-known models in this stream include the technology adoption lifecycle model (see Rogers 1962), the Bass diffusion model (Bass 1969), and the technology acceptance model (TAM; see Davis 1989, Bagozzi et al. 1992). The second stream analyzes technology adoption from the perspective of social planning instead of from the perspective of individuals. In this manuscript, systematic technology adoption refers to the second stream, and such technology adoption is planned by social planners on a system level to meet a certain system objective, e.g., to satisfy a country's demand for electricity at a minimum total cost and with acceptable environmental effects.

Significant effort has been devoted to the development of operational optimization models of systematic technology adoption. Well-known examples of such models include MESSAGE (Messner and Strubegger 1994) and MARKAL (Seebregts 2001). The purpose of these models is usually to determine the optimal systematic technology adoption to minimize the total cost of the entire system subject to various constraints. To date, most of these models assume the existence of a global social planner and ignore the existence of heterogeneous decision makers who interact with one another in the system under study. Although certain optimization models involve different regions (e.g., Messner and Strubegger 1994) and quite a few of such models introduce elements such as different hurdle rates for different sectors (e.g. Dodds 2014) that imitate differences in the preferences of specific representative agents, existing models commonly ignore uncertain technological learning, the heterogeneity of agents in terms of their limited foresight and risk attitudes, and especially the interactions among agents.

This study develops a stylized (or conceptual) optimization model of systematic technology adoption with heterogeneous agents (i.e., decision makers) and uncertain technological learning. Technological learning denotes the cost reduction of new technologies while experience with them accumulates (Arrow 1962); this reduction is a classic example of increasing returns (Arthur 1989), which has been ignored in most existing operational optimization models. In the model presented in this manuscript, systematic technology adoption is no longer decided by a global social planner. Instead, there are many agents in the system, and each of them attempts to optimize technology adoption for part of the entire system. The agents are heterogeneous in terms of their foresights and attitudes toward the risk resulted from overestimating technological learning. And there is technological spillover among agents, which means that the technology experience one agent gains can somehow benefit other agents. Using this model, we explore how the agents' heterogeneities (in foresights and risk attitudes) and technological spillover affect the optimal solutions of systematic technology adoption.

Some researchers (e.g Krusell and Smith 2010) have argued that a well calibrated representative model (i.e., a global-social-planner model) can perform well enough when the interest is placed on aggregate variables such as the overall adoption of a new technology. In our research, we show that it is possible that a representative model can be recalibrated to result in the same adoption rate of a new 
technology as that resulted from a multi-agent model. But when implementing a carbon tax in the system, it is possible that the representative model fails to adjust, i.e., the adoption rate is different from that with the multi-agent model. This experiment enhances the idea that it makes sense to do modeling considering heterogeneous agents.

The model presented in this manuscript is not intended by any means to be a "realistic" model in the sense of showing technological or sectoral detail. Rather, the model is primarily intended to be used for exploratory modeling purposes and as a heuristic research device for the in-depth examination of the effects of alternative model formulations on the dynamics of endogenous technology transitions.

The rest of the manuscript is organized as follows. Section 2 introduces the framework of operational optimization models of systematic technology adoption and the heterogeneous agents in our model. Section 3 presents the stylized optimization model of systematic technology adoption with heterogeneous agents and uncertain technological learning. Section 4 presents the experiment which shows that a recalibrated representative model reacts differently from the multi-agent model when a carbon tax is implemented in the system. Section 5 explores how the agents' heterogeneities and interactions affect systematic technology adoption. Section 6 presents the conclusions.

\section{Modeling Framework with Heterogeneous Agents}

\subsection{Framework of optimization models of systematic technology adoption}

Fig. 1 presents an illustration of optimization models of systematic technology adoption (Ma 2010). The right side of Fig. 1 is the list of human demands, such as heating and transportation. The left side is the list of natural resources, such as coal and gas. In the middle, there are chains of technologies that link natural resources to human demands. For example, Tech 1 is coal mining, Tech 2 is a coal power plant, which can generate electricity with the output of Tech 1 , and the output of Tech 2 can subsequently be used as the input for other technologies to satisfy human demands.

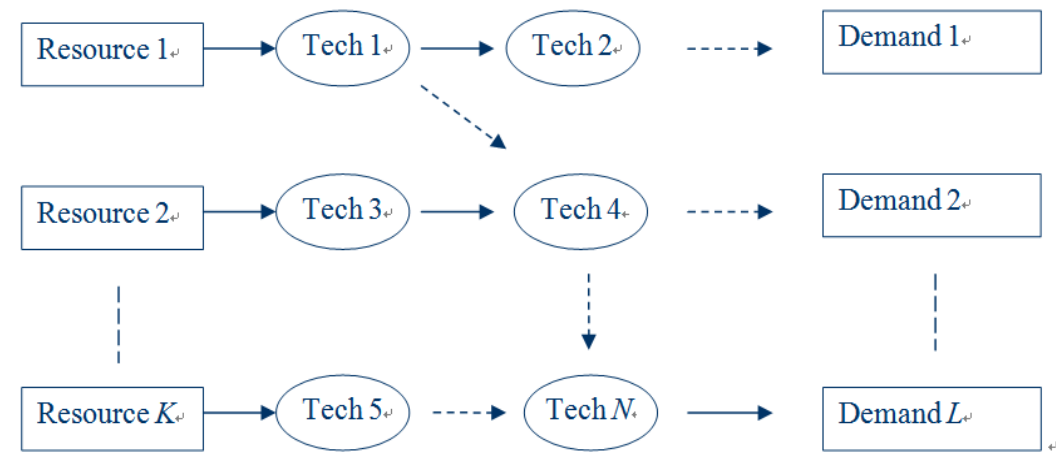

Figure 1. Framework of optimization models of systematic technology adoption 
Technologies in Fig. 1 include both mature existing technologies such as traditional coal power plants and new technologies such as photovoltaic power plants. With the system structure presented in Fig. 1, the objective of optimization models of systematic technology adoption is to find the optimal combinations of technologies along the time dimension so that the total cost of the system is minimized with various constraints (e.g., demands should be satisfied).

\subsection{Heterogeneous agents and technological spillover effect}

Most existing optimization models of systematic technology adoption assume the existence of a global social planner, who decides the technology adoption for the entire system, as illustrated on the left side of Fig. 2. The stylized model that will be presented in Section 3 assumes that the entire system is managed by a number of agents, abbreviated as A1, A2 ..., as shown on the right side of Fig. 2. Each agent makes decisions for a portion of the entire system. These agents are heterogeneous in terms of their lengths of foresight and attitudes toward the risk that results from the uncertainty of technological learning, and there are interactions among agents in terms of technological spillover. We provide more explanations on the notions of foresight of agents, uncertain technological learning, and technological spillover in the following.
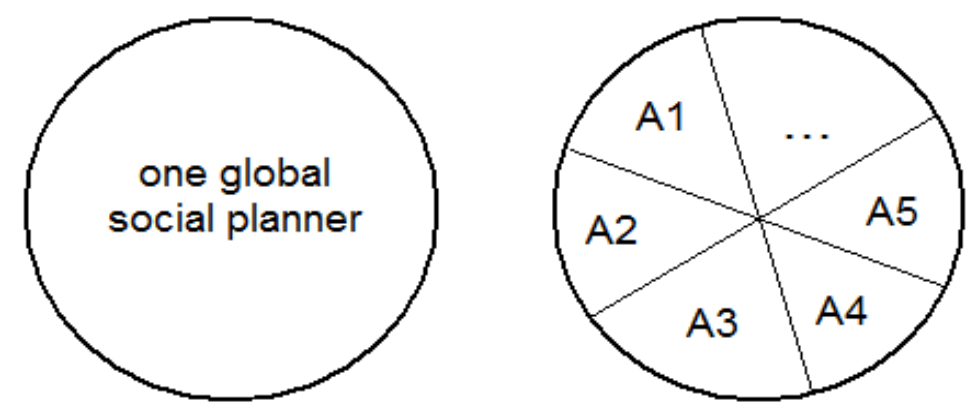

Figure 2. One global social planner versus heterogeneous agents

\section{a) Foresight of agents}

Traditional optimization models of systematic technology adoption usually assume a decision maker who has complete information about the future, i.e., with a perfect foresight for a long period of time (e.g., see Azar et al. 2003; Barreto and Kypreos 2002). In reality, decision makers commonly do not have perfect foresight and need to adjust decisions from time to time. In recent years, researchers have begun to introduce limited foresight into optimization models of systematic technology adoption (e.g., Hedenus et al. 2006; Martinsen et al. 2007; Keppo and Strubegger 2010; and Chen and Ma 2014).

Fig. 3 illustrates perfect foresight and two types of limited foresight schemes (Keppo and Strubegger 2010). From Fig. 3, we can see that the entire time horizon is composed of only one decision period with perfect foresight, while it is divided into several connecting decision periods with 
limited foresight. With the first type of limited foresight, there is no overlap between two connecting periods, which means a decision maker will adjust his/her decisions at the end of a decision period; and with the second type of limited foresight, there is an overlap between two connecting decision periods, which means that a decision maker will adjust his/her decisions before the end of a decision period. In Fig. 3, a decision interval is a basic time unit for installing new technological capacities, and each decision period is composed of several decision intervals.

The stylized model that will be presented in Section 3 assume that agents have different lengths of (limited) foresight, and the model adopts the second type of limited foresight (i.e., the LF2 in Fig.3). Each agent makes decisions every 10 years, there are overlaps between connecting decision periods, and the longer the agent's foresight is, the longer the overlap is.

With technological learning, the optimization model will be non-linear and non-convex. With limited foresight, a decision maker is myopic, which means he/she focuses only on minimizing the cost in the current decision period. At each decision period, there could be more than one local optimal solution to technology adoption with highly similar total costs but different technology adoption paths (Ma 2010, Chi et. al 2012). In this manuscript, for the sake of simplification, each agent selects the local optimal solution with the minimal cost at each decision period.

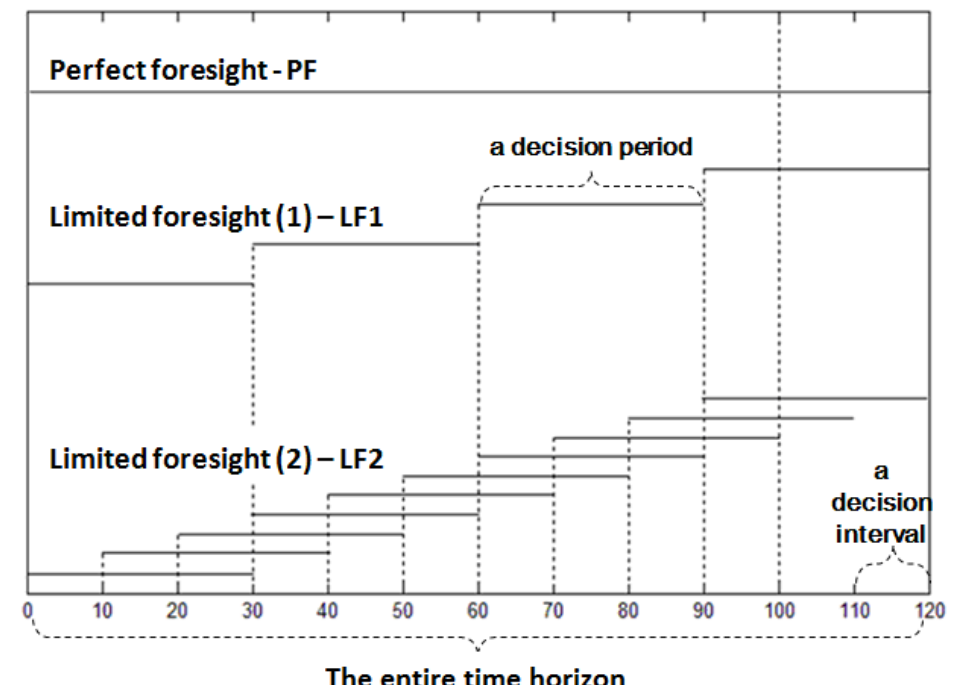

Figure 3. Perfect foresight and two types of limited foresight schemes

\section{b) Uncertain technological learning and agents' risk attitudes}

Technological learning denotes the cost reduction of new technologies while experience with them accumulates (Arrow 1962). With technological learning, the cost of using a new technology is a function of previous decisions on investment on the new technology. Technological learning has been believed to be an endogenous driving force for adopting advanced but currently expensive technologies (e.g., Schwoon 2008). Historical observations show that technological learning is quite uncertain and learning rates commonly follow a lognormal distribution (McDonald and 
Schrattenholzer 2001). When making decisions on adopting new technologies, overestimating technological learning could result in higher cost of using new technologies than what is expected

The model that will be presented in Section 3 assumes there is technological learning for new technologies, and the learning rates could be uncertain. Each agent in the model has different attitudes toward the risk resulting from uncertain technological learning. Agents in the model treat the risk as a penalty to their objective functions and different agent implements a different weight (denoting risk attitude) on the risk.

\section{c) Technological spillover among agents}

With different foresights and different risk attitudes, different agents will adopt a new technology at different times, which means some agents will invest in the new technology earlier than others will. In the model presented in Section 3, we assume earlier adopters' experience on the new technology could spill over to followers to some extent, which means followers could face a lower initial cost of using the new technology than earlier adopters.

\section{The Model}

For the sake of transparency, the techno-economic system of our model is deliberately kept simple. The simplification also follows previous research on endogenous technological change models (e.g., see Chen and Ma 2014, Ma and Nakamori 2009; Grübler and Gritevskyi 1998; Manne and Barreto 2004). In the model, the economy demands one homogenous good, e.g., electricity, and the demand increases over time. There are three types of technologies that can be used to produce the goods. The first is called existing technology, or simply T1, which has low efficiency and a low initial investment cost. The second is called incremental technology, or T2, which has higher efficiency and a higher initial investment cost. The third one is called revolutionary technology, or T3, which has the highest efficiency and the highest initial investment cost. T2 and T3 have learning potential; that is, the cost of using them tends to decrease as experience with them accumulates.

Although the model is conceptual, its framework and main assumptions follow existing operational optimization models, such as the MESSAGE model (Messner and Strubegger 1994) and the MARKAL model (Seebregts 2001). The mathematical formulations introduced in the following also follow previous literature on conceptual models of endogenous technological change (e.g., see Grubler and Gritsevskyi 1998; Manne and Barreto 2004; Ma 2010; Chen and Ma 2014). In short, the formulations used in our model were primarily obtained from the existing literature and studies. The novelty of the model is the introduction of heterogeneous agents who make decisions simultaneously in the system, and there is technological spillover among them.

We assume that there are $N$ heterogeneous decision agents in the system, and each of the agents attempts to identify the optimal technology adoption strategy for a portion of the system with the optimization model described in Eqs. (1) to (14). 
For agent $n(n=1, \cdots, N)$, its own cumulative experience with technology $i(i=1,2,3)$ at time $t$ is calculated as its cumulative production with technology $i$ by time $t$, as shown in Eq. (1).

$$
\bar{x}_{i, n}^{t}=\sum_{j=1}^{t} x_{i, n}^{j}+\bar{x}_{i, n}^{0}
$$

where $x_{i, n}^{j}$ denotes agent $n$ 's production using technology $i$ at time $j, \bar{x}_{i, n}^{0}$ denotes agent $n$ 's cumulative production using technology $i$ before the first decision period, and $\bar{x}_{i, n}^{t}$ denotes agent $n$ 's cumulative production using technology $i$ by time $t$. In the model, the existing technology does not have technological learning potential, so we do not need to use Eq.(1) to calculate $\bar{x}_{i, n}^{t}$ when $i=1$. The incremental technology and revolutionary technology are treated as new technologies, they do not have cumulative production before the first decision period, that is to say $\bar{x}_{i, n}^{0}=0$ when $i=2,3$.

At each decision period, each agent's production with technology $i$ cannot beyond its total installed capacity which is calculated with Eq. (2).

$$
\bar{y}_{i, n}^{t}=\left\{\begin{array}{cc}
\sum_{h=t-\tau_{i}}^{t} y_{i, n}^{h} & t>\tau_{i} \\
\sum_{h=1}^{t} y_{i, n}^{h}+\frac{\tau_{i}-t}{\tau_{i}} \bar{y}_{i, n}^{0} & t \leq \tau_{i}
\end{array},\right.
$$

where $\bar{y}_{i, n}^{t}$ denotes agent $n$ 's total installed capacity of technology $i$ at time $t, \tau_{i}$ denotes the plant life of technology $i, y_{i, n}^{h}$ denotes agent $n$ 's newly installed capacity of technology $i$ at time $h$, and $\bar{y}_{i, n}^{0}$ denotes agent $n$ 's initial total installed capacity of technology $i$ before the first decision period. The model assumes before the first decision period, all demand is satisfied by using the existing technology, its installed capacity distributed evenly in age, and thus $\frac{\tau_{i}-t}{\tau_{i}} \bar{y}_{i, n}^{0}$ denotes the remaining part of the initial total installed capacity. The incremental technology and the revolution technology are treated as new technologies, the model assumes there are no installed capacity of them before the first decision period thus $\bar{y}_{i, n}^{0}=0$ when $i=2,3$.

There is a technological spillover effect (or technological 'free-riding') among agents, which means that one agent's accumulated experience with a new technology could benefit other agents. Agent $n$ 's total experience with (or knowledge of) technology $i$ at time $t$ is the sum of its own experience and the experience spillover to it from other agents, as shown in Eq. (3):

$$
\hat{x}_{i, n}^{t}=\bar{x}_{i, n}^{t}+\theta \sum_{m=1}^{100} \bar{x}_{i, m}^{t}(m \neq n, \theta \in[0,1] \text { and } i=1,2,3)
$$


where $\bar{x}_{i, n}^{t}$ denotes agent $n$ 's own cumulative experience with technology $i$ at time $t$, which resulted from agent $n$ 's previous investment in technology $i$, as shown in Eqs. (1)-(2); $\theta \sum_{m=1}^{100} \bar{x}_{i, m}^{t}(m \neq n)$ is the spillover from other agents' experience with technology $i$; $\theta \in[0,1]$ is the spillover rate, and the smaller it is, the weaker the spillover effect is; and $\hat{x}_{i, n}^{t}$ denotes agent $n$ 's total experience with technology $i$ by time $t$.

The existing technology has no learning potential and its unit investment cost is constant in the model. The incremental and the revolutionary technologies have learning potential and their unit investment costs will decrease with their cumulative productions. With the technological spillover effect for agent $n$, its unit investment cost for technology $i$ at time $t$ is calculated with Eq. (4):

$$
C F_{i, n}^{t}=\left\{\begin{array}{cc}
C F_{i, n}^{0} & (i=1) \\
C F_{i, n}^{0} \times\left(\hat{x}_{i, n}^{t-1} / \hat{x}_{i, n}^{0}\right)^{-b_{i}} & (i=2,3)
\end{array}\right.
$$

where $b_{i}$ is the elasticity of the investment cost with regard to the cumulative installed capacity, and it is easy to show that $1-2^{-b_{i}}$ (which is called the learning rate of technology $i$ ) is the percentage of the decrease in the investment cost when the total experience doubles. In addition, $C F_{i, n}^{0}$ denotes agent $n$ 's initial unit investment cost for technology i. $\hat{x}_{i, n}^{0}$ denotes agent $n$ 's initial cumulative production with technology $i$. The incremental and the revolutionary technologies are treated as new to the system, and $C F_{i, n}^{0}(i=2,3)$ denotes the unit investment cost of the first unit, in other words, $\hat{X}_{i, n}^{0}(i=2,3)$ equals to 1 in Eq. 4 and thus the formulation could be simplified to $C F_{i, n}^{t}=C F_{i, n}^{0} \times\left(\hat{X}_{i, n}^{t-1}\right)^{-b_{i}}$ when $i=2,3$.

Agent $n$ 's cost of extracting each unit resource at time $t$, which is denoted by $C_{E, n}^{t}$, is a linear function of resource depletion and is calculated using Eq. (5):

$$
C_{E, n}^{t}=C_{E, n}^{0}+k_{E}{\overline{R_{n}}}^{\mathrm{t}} \text {, }
$$

where $C_{E, n}^{0}$ denotes agent $n$ 's initial cost of extracting each unit resource at time $t$, coefficient $k_{E}$ denotes the sensitivity of the extraction cost to cumulative extraction, and $\bar{R}_{n}$ t denotes agent $n$ 's cumulative extraction by time $t$, which is calculated with Eq. (6):

$$
{\overline{R_{n}}}^{\mathrm{t}}=\sum_{j=1}^{\mathrm{t}} R_{n}^{j},
$$

where $R_{n}^{j}$ denotes agent $n$ 's total resource consumption at time $j$, which is the sum of the resources consumed by all of agent $n$ 's technologies at time $j$, as shown in Eq. (7): 


$$
R_{n}^{j}=\sum_{i=1}^{3} \frac{1}{\eta_{i}} x_{i, n}^{j},
$$

where $\eta_{i}$ denotes the efficiency of technology $i$, and $x_{i, n}^{j}$ denotes agent $n$ 's production using technology $i$ at time $j$.

The demand of each agent increases over time with a constant annual growth rate, as shown in Eq. (8):

$$
D_{n}^{t}=D_{n}^{0}(1+\alpha)^{t}
$$

where $D_{n}^{t}$ denotes agent $n$ 's total demand at time $t, D_{n}^{0}$ denotes agent $n$ 's initial demand, and $\alpha$ denotes the annual growth rate of the demand.

With the assumption of deterministic learning, each agent attempts to minimize its total cost, which is denoted using Eq. (9):

$$
\min \sum_{i=1}^{3} \sum_{t=1}^{T_{n}} \frac{1}{(1+\delta)^{t}} C F_{i, n}^{t} y_{i, n}^{t}+\sum_{t=1}^{T_{n}} \frac{1}{(1+\delta)^{t}} C_{E, n}^{t} R_{n}^{t}+\sum_{i=1}^{3} \sum_{t=1}^{T_{n}} \frac{1}{(1+\delta)^{t}} C_{O M i} x_{i, n}^{t},
$$

where $C_{\mathrm{OMi}}$ denotes the operation and maintenance $(\mathrm{O}+\mathrm{M})$ cost of each technology $i, \delta$ is the discount rate, and $T_{n}$ denotes the number of decision intervals within one of agent $n$ 's decision period.

The constraints of the optimization model are included in Eqs. (10) to (13):

$$
\begin{array}{ll}
D_{n}^{t} \leq \sum_{i=1}^{3} x_{i, n}^{t} & \left(\mathrm{t}=1, \ldots T_{n}\right)(\mathrm{n}=1, \ldots, N) \\
x_{i, n}^{t} \leq \bar{y}_{i, n}^{t} & \left(\mathrm{t}=1, \ldots T_{n}\right)(\mathrm{i}=1,2,3)(\mathrm{n}=1, \ldots, N) \\
x_{i, n}^{t} \geq 0 & \left(\mathrm{t}=1, \ldots T_{n}\right)(\mathrm{i}=1,2,3)(\mathrm{n}=1, \ldots, N) \\
y_{i, n}^{t} \geq 0 & \left(\mathrm{t}=1, \ldots T_{n}\right)(\mathrm{i}=1,2,3)(\mathrm{n}=1, \ldots, N)
\end{array}
$$

The constraint function (10) indicates that at each time $t$, for each agent, the total production of all three types of technologies must satisfy the agent's demand. Constraint function (11) denotes that the production for each agent using technology $i$ at time $t$ cannot go beyond the total installed capacity of technology $i$ at time $t$. Constraint functions (12) and (13) indicate that all decision variables cannot be negative.

With uncertain technological learning, the objective function of each agent can be written as Eq. (14):

$$
\begin{aligned}
& \min \sum_{i=1}^{3} \sum_{t=1}^{T_{n}} \frac{1}{(1+\delta)^{t}} C F_{i, n}^{t} y_{i, n}^{t}+\sum_{t=1}^{T_{n}} \frac{1}{(1+\delta)^{t}} C_{E, n}^{t} R_{n}{ }^{t}+\sum_{i=1}^{3} \sum_{t=1}^{T_{n}} \frac{1}{(1+\delta)^{t}} C_{\text {OMi }} x_{i, n}^{t}, \\
& +\rho_{n}\left\{\mathrm{E}\left\{\sum_{t=1}^{T_{n}} \max \left\{0,\left[C F_{3, n}^{t}(\psi)-C F_{3, n}^{t}\right] y_{3, n}^{t}\right\}\right\}\right\}
\end{aligned}
$$

The first line of objective function (14) is the same as that of objective function (9), and in the 
second line, $\rho_{n}$ denotes agent $n$ 's attitude toward risk (a larger $\rho_{n}$ indicates a more risk-averse attitude). E denotes expectation, and $C F_{3, n}^{t}(\psi)$ is a random variable; here, $\psi$ denotes an element from a probability space characterized by a log-normal distribution. The constraints remain the same as in the constraint functions (10) to (13).

With Eq. (14), the model is a stochastic optimization problem. It is solved for a sufficiently large sample $M$, where the size of $M$ is determined through successive experiments. Several successive model runs with the same sample size $M$ are compared. If no major changes in the solution structure and the objective function can be observed, $M$ is considered sufficiently large (For more detail, see Messner et al. 1996).

Using the model introduced above, this manuscript focuses primarily on the agents' heterogeneities as follows:

- The agents' foresight could be different; i.e., the length of their decision periods (see Fig. 3) could be different.

- When uncertainties in technological learning are considered, agents may have different risk attitudes; i.e., for different agents, the $\rho_{n}$ in Eq. (14) could be different.

With the above heterogeneities, different agents could have different optimal solutions for adopting new technologies (T2 and T3 in the model), and with the technological spillover effect among agents, the agents who adopt new technologies relatively late can benefit from those who adopt the new technologies early. In the following section, we will explore how the heterogeneities and technological spillover effect influence the adoption of new technologies at the system level.

\section{A calibrated representative model VS the multi-agent model}

Some researchers (e.g Krusell and Smith 2010) have argued that a well calibrated representative model (i.e., a global-social-planner model) can perform well enough when the interest is placed on aggregate variables. Then does it still make sense to model agents' heterogeneities and their interactions? For answering this question, we design an experiment in which a representative model is recalibrated to result in the same adoption rate of the new technology T3 as that resulted from the multi-agent model introduced in section 3. Then we implement a carbon tax in both the representative model and the multi-agent model. Our experiment shows that it is possible that the representative model and the multi-agent model react differently to the same carbon tax. 


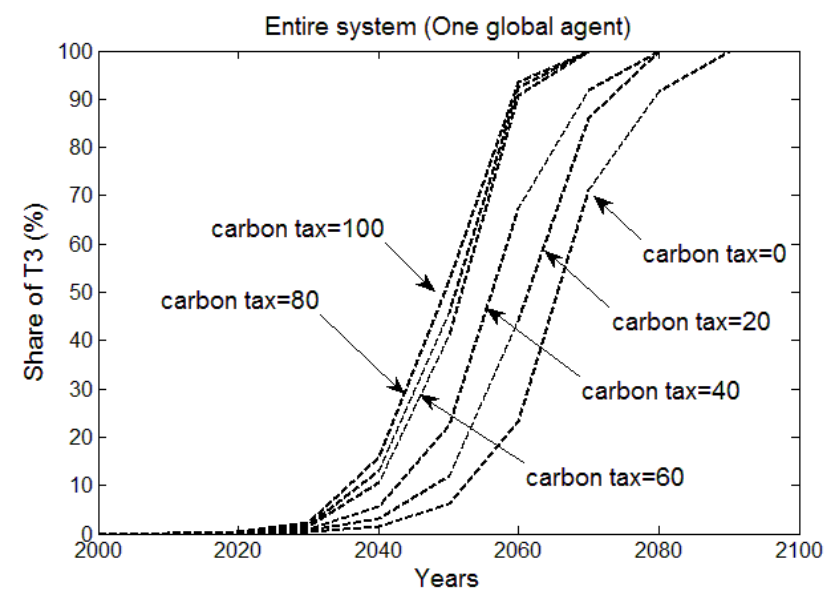

Figure 4. Adoption of $\mathrm{T} 3$ with the calibrated representative model

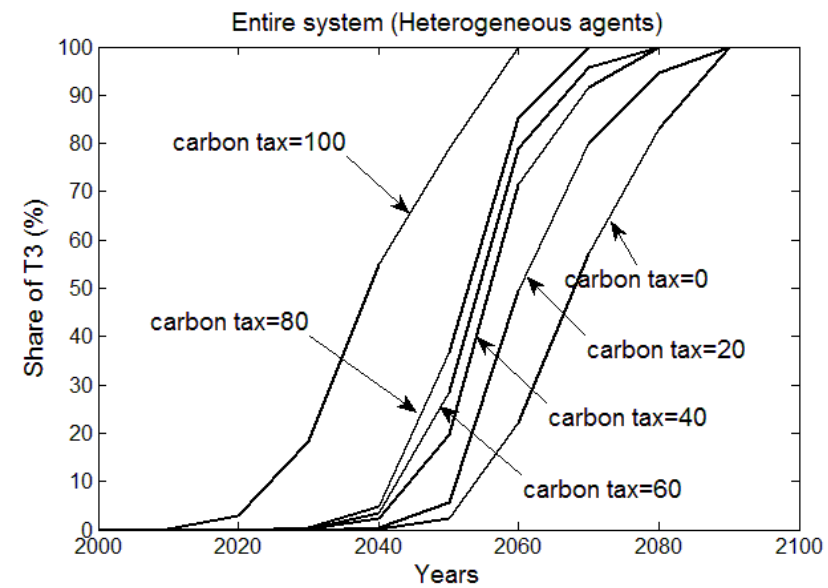

Figure 5. Adoption of T3 with the multi-agent model

In the experiment, the representative model assumes a global social planner plans the adoption of T3 for the entire system for the period from 2000 to 2100; the multi-agent model assumes the entire system is divided into 10 portions with the same size, there are 10 agents and each of them plans the adoption of T3 for one portion. These 10 agents have different risk attitudes ( $\rho_{n}$ in Eq. 14) ranging from 0.1 to 1 . Each of them makes 10 sequential decisions from 2000 to 2100 with each decision period as 50 years, and there is a 40-year overlap between two connecting decision periods. The technological spillover rate ( $\theta$ in Eq. 3 ) among agents is assumed to be $1 \%$. The representative model is recalibrated so that in its optimal solution, the adoption of T3 in the entire system is similar to that resulted from the multi-agent model. The recalibration is done by adjusting the learning rate of T3 since previous studies (i.e., Grübler \& Gritsevskyi 1998) have shown learning rates are critical parameters for endogenous technological change models. For the multi-agent model, the learning rate of T3 is assumed to be $35 \%$, and the learning rate in the representative model is recalibrated to 18.38\%. Other parameter values can be found in Table 1.

The experiment does not aim to replicate any reality. The main purpose of the experiment is to see whether a well recalibrated representative model will react to a carbon tax differently from the 
multi-agent model. Fig. 4 plots the adoption of T3 with the recalibrated representative model, and Fig. 5 plots the adoption of T3 with the multi-agent model. As we can see, when there is no carbon tax (denoted as carbon tax $=0$ in Fig.4 and Fig. 5), the adoption of T3 with the recalibrated representative model and the multi-agent model is similar. When a carbon tax is implemented, it is possible that the two models react to it differently. When the carbon tax is 100 US\$/TC, with the calibrated representative model, the adoption of T3 is lagged for 10-20 years than that with the multi-agent model. This experiment enhances the idea that although a well recalibrated representative model can perform well enough when the interest is placed on aggregate variables, it still makes sense to model agents' heterogeneities and their interactions.

\section{Simulations and Analysis}

\subsection{Parameter values in simulations}

Table 1. Initial values of the parameters for agents ( $\forall n \in[1,100])$

\begin{tabular}{lccc}
\hline Parameters & $\begin{array}{c}\text { Existing } \\
\text { technology }\end{array}$ & $\begin{array}{c}\text { Incremental } \\
\text { technology }\end{array}$ & $\begin{array}{c}\text { Revolutionary } \\
\text { technology }\end{array}$ \\
\hline Initial investment cost (US\$/kW) & $C_{1, n}^{0}=1000$ & $C F_{2, n}^{0}=2000$ & $C F_{3, n}^{0}=30000$ \\
Efficiency & $\eta_{1}=30 \%$ & $\eta_{2}=40 \%$ & $\eta_{3}=90 \%$ \\
Plant life (year) & $\tau_{1}=30$ & $\tau_{2}=30$ & $\tau_{3}=30$ \\
Initial total installed capacity (kW) & $\bar{y}_{1, n}^{0}=100$ & $\bar{y}_{2, n}^{0}=0$ & $\bar{y}_{3, n}^{0}=0$ \\
O+M cost (US $\$ \mathrm{~kW}$-year) & $C_{O M 1}=30$ & $C_{O M 2}=50$ & $C_{O M 3}=50$ \\
\hline Learning rates & $(\mathrm{No} \mathrm{learning)}$ & $1-2^{-b_{2}}=10 \%$ & $1-2^{-b_{3}}=30 \%$ \\
\hline Other parameters & & & \\
\hline Initial demand & & $D_{n}^{0}=100$ & \\
Annual growth rate of demand & & $\alpha=2.6 \%$ & \\
Initial extracting cost (US\$/kW) & & $C_{E, n}^{0}=200$ & \\
Extraction cost coefficient & & $k_{E}=0.01$ & \\
Discount rate & & $\delta=5 \%$ & \\
Number of agents & & $N=100$ & \\
\hline
\end{tabular}

To explore how technology adoption is influenced by the heterogeneity of the agents' limited foresight and risk attitudes and the technological spillover effect among them, our simulations assume that agents make decisions with the same demand dynamics, resource depletion dynamics, and 
technological learning rates. We assume that 100 agents are making decisions regarding technology adoption simultaneously. Table 1 gives the initial values of the parameters for each agent in our simulations.

With the model, we first explore how agents' heterogeneous foresights influence the adoption of the advanced technology T3 (in subsection 5.2), then explore how agents' heterogeneous risk attitudes influence the adoption of T3 (in subsection 5.3), and then analyze the adoption of T3 with both heterogeneous foresights and heterogeneous risk attitudes (in subsection 5.4), and finally analyze the adoption of T3 with different technological spillover rates (in subsection 5.5).

\subsection{Technology adoption with heterogeneous foresights}

We explore how agents' different foresights influence the adoption of T3 by comparing simulations with agents having the same foresight with simulations with agents' foresights as random values from the set $\{20,30,40,50,60,70,80,90\}$. In all of the simulations presented in this subsection, all of the agents make decisions with deterministic learning rates, i.e., each agent's objective function is denoted using Eq. (9); and when there is technological spillover effect, the technological spillover rate $(\theta)$ is set to 0.5 . (We will explore how different technological spillover rates influence the adoption of T3 later.)

Fig. 6 plots the adoption of T3 in the entire system when all 100 agents have the same foresight. In Fig. 6, the numbers on the lines denote the agents' foresight. The left part of Fig. 6 shows the results obtained when there is no technological spillover effect among the agents, and the right part present the results when there is a technological spillover effect. When all of the agents have the same foresight, each agent's adoption of T3 is the same as the adoption of T3 in the entire system because all of the agents are the same. Thus, the lines shown in Fig. 6 can also be viewed as each agent's adoption of T3. In general, there is a tendency for a delay in the adoption of T3 when the agents' foresight is shorter. The comparison of the left and right parts of Fig. 6 revealed that the addition of a technological spillover effect most of time will bring forward the adoption of T3. But there are exceptions. The adoption of T3 is postponed a little bit when all of the agents' foresights are 40 years. This is because adding a technological spillover effect will strengthen the lock-in of T2 with a stronger learning effect. In most cases, this lock-in effect is not adequate for postponing the adoption of T3. However, when all of the agents' foresights are 40 years, the adoption of T3 is postponed a little bit. 

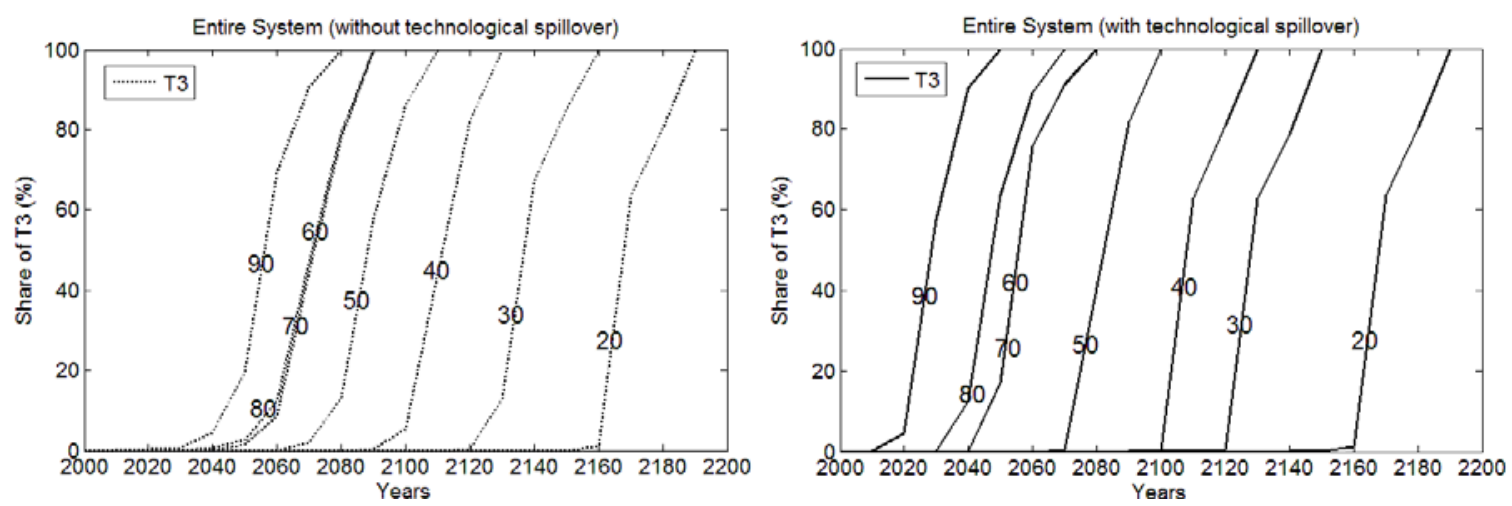

Figure 6: Adoption of T3 in the entire system with agents having the same foresight
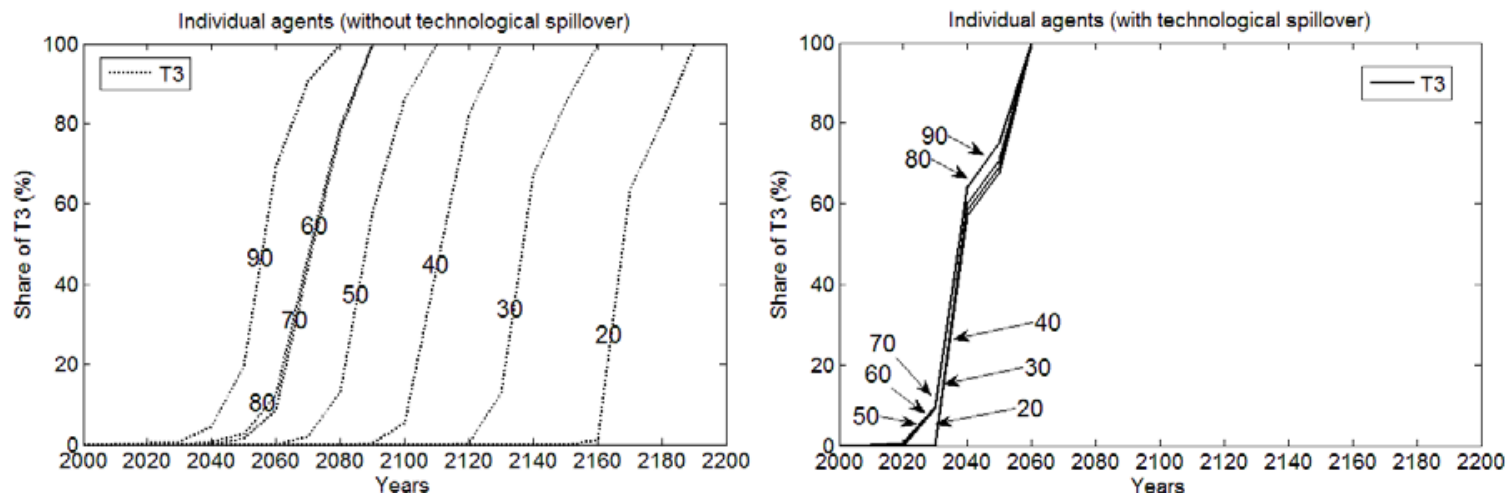

Figure 7. Adoption of T3 by individual agents with different foresight

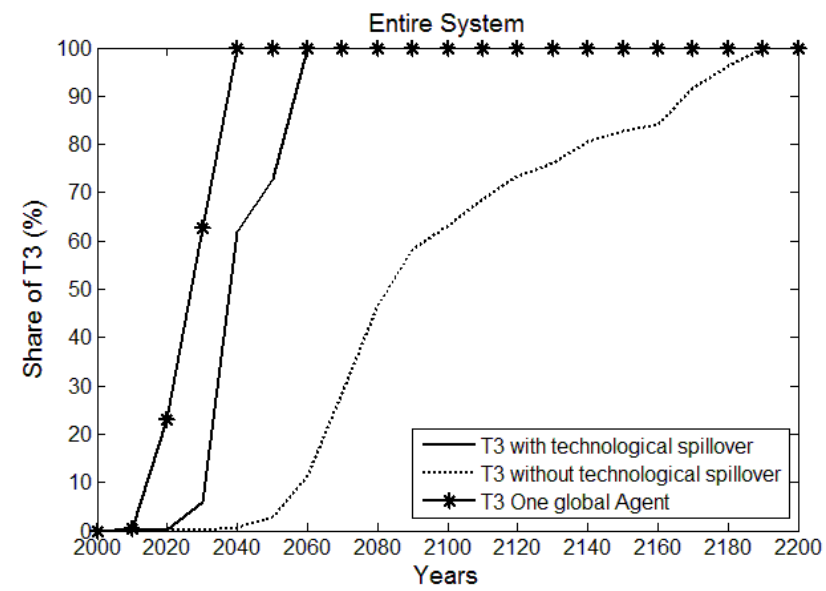

Figure 8. Adoption of T3 in the entire system with heterogeneity in the agents' foresight

Fig. 7 plots the adoption of T3 by different agents when their foresights are assigned random values from the set $\{20,30,40,50,60,70,80,90\}$, with the numbers on the lines representing the agents' foresights. The left part of Fig. 7 plots the agents' adoption of T3 when there is no technological spillover effect, and the right part plots the adoption of T3 when there is technological spillover. The left parts of Fig. 6 and Fig. 7 look the same. However, the lines on the left part of Fig. 6 are the results of all agents in different simulations, whereas the lines on the left part of Fig. 7 are the 
results of different agents in one simulation. They look the same because when there is no technological spillover effect, each agent operates independently, and thus, agents that have the same foresight will have the same results. The right part of Fig. 7 shows that when there is a technological spillover effect, T3 appears at almost the same time for agents with different foresight (around the year 2040).

Fig. 8 plots the adoption of T3 in the entire system with and without a technological spillover effect when agents have different foresight, from which we can see that T3 dominates the entire system about 130 years earlier when there is a technological spillover effect than when there is no technological spillover effect. Fig. 8 also plots the adoption of T3 when there is a global social planner. As we can see, with a global social planner, the adoption of T3 is much earlier and faster than in situations in which there are 100 agents making decisions simultaneously. This is because the entire system composed of 100 agents is divided into 100 portions. Thus, each agent faces a small market that is not sufficiently large to cover the high investment cost required for the development of T3, and the rational decision is to postpone the adoption of T3.

Fig. 9 plots the differences in the year T3 appears for agents with different foresight in cases in which there is and is not a technological spillover effect. As observed in Fig. 9, the longer an agent's foresight is, the smaller the difference of the year in which T3 appears is. This finding suggests that technological spillover has a larger effect on agents with shorter foresight, which allows them to change their decisions in a timelier manner; in other words, they are more adaptive and thus can benefit more from other agents' accumulated experience when there is a technological spillover effect.

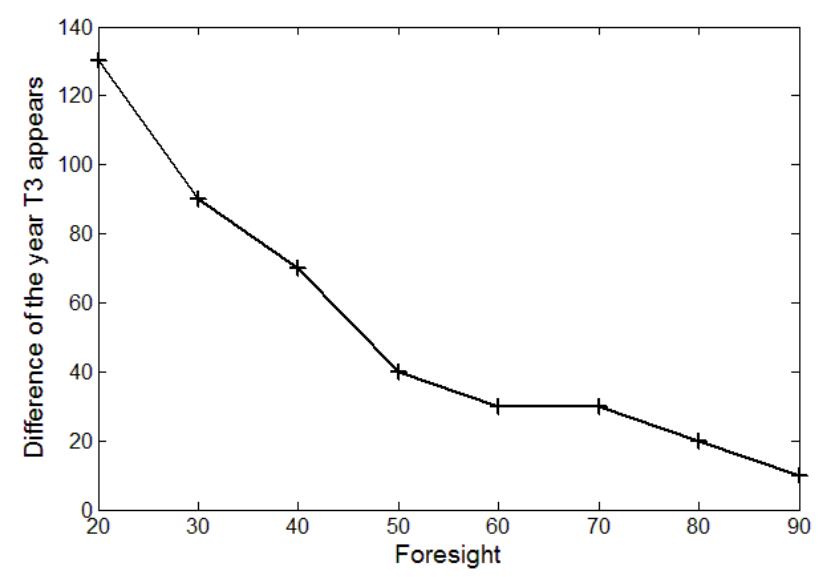

Figure 9. Difference in the appearance of T3 for agents with different foresight in the absence and presence of a spillover effect

For optimization models of systematic technology adoption, the above simulations show that the assumption of the existence of multiple decision makers implies a slower adoption of advanced technologies than that obtained with the assumption of the existence of a global social planner. Since 
shorter foresight generally leads to later adoption of a new technology, as illustrated in Fig. 6 and Fig. 7, a technological spillover effect can help accelerate the adoption of advanced technologies when agents have different foresights, and thus, followers can benefit from adjusting their decisions. These results are in accordance with people's intuition. The main contribution of our simulations is verifying this intuition through the generation and analysis of optimization results.

\subsection{Technology adoption with heterogeneous risk attitudes}

To explore how agents' heterogeneous risk attitudes influence the adoption of T3, we run simulations with each agent's risk attitude as a random value following a normal distribution. In each simulation presented in this subsection, all of the agents have the same foresight and make decisions with uncertain learning rates, i.e., each agent's objective function is denoted based on Eq. (14). Each agent's risk attitude is given a random value following a normal distribution $N\left(\mu, \sigma^{2}\right)$, where $\mu$ is the mean of the distribution, and $\sigma$ is the standard deviation. When there is a technological spillover effect, the technological spillover rate $(\theta)$ is set to 0.5 .
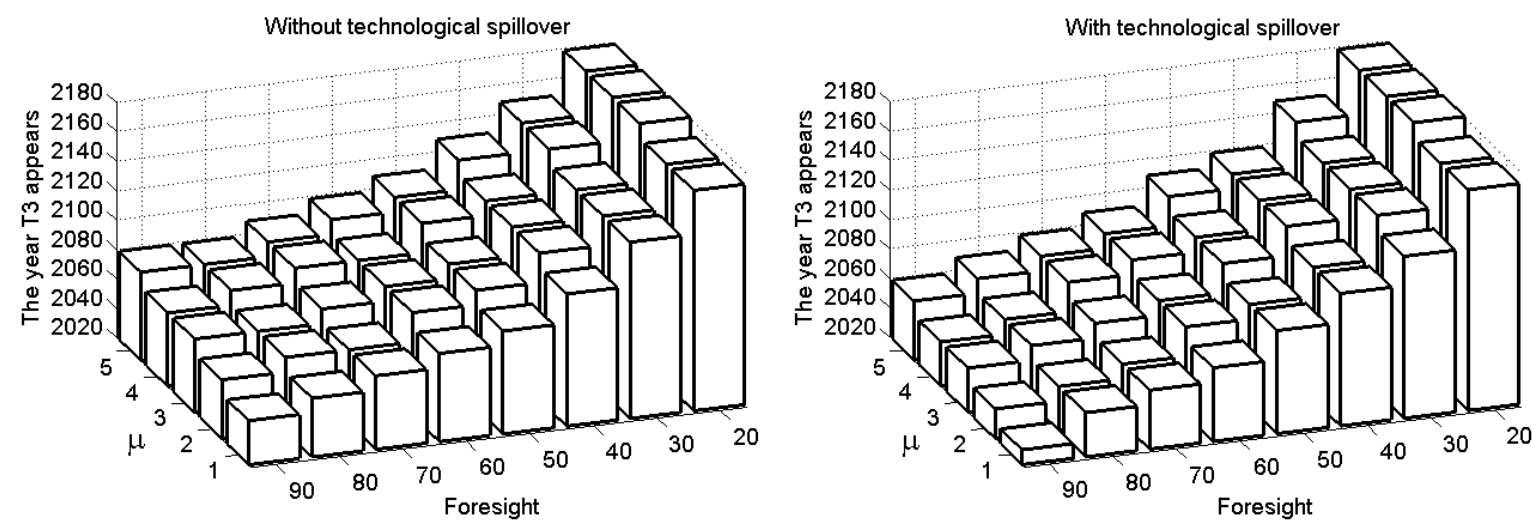

Figure 10. Years in which T3 appears in the entire system with different combinations of foresight and mean attitudes (with $\sigma=0.5$ )

Fig. 10 shows the years in which T3 appears in the entire system with different combinations of agents' foresight and mean risk attitudes and with $\sigma=0.5$. The left part of Fig. 10 shows the years in which T3 appears in the absence of a technological spillover effect, and the right part shows the years when there is a technological spillover effect. As shown in Fig. 10, as the agents' mean risk attitude $\mu$ becomes larger, the year in which T3 appears in the entire system generally becomes later, and the technological spillover effect could bring forward the years T3 appear in the system, especially when agents' foresight is comparatively longer (when the agents' foresight is 80 or 90 ). 

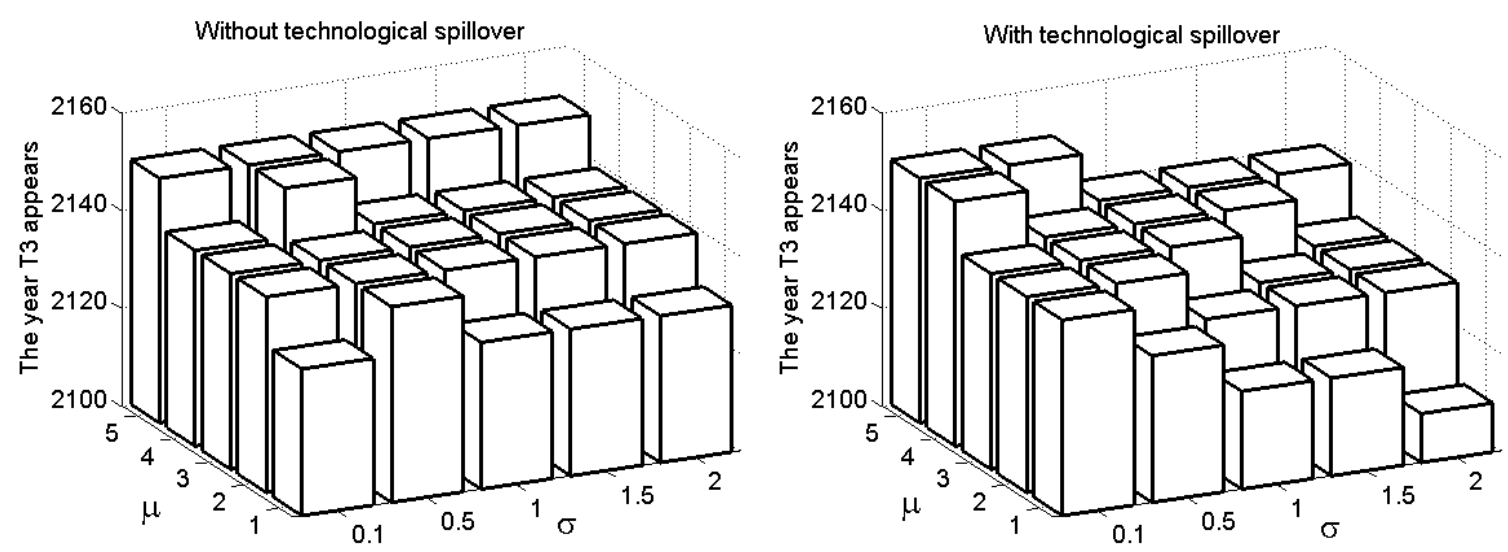

Figure 11. Years in which T3 appears in the entire system with different combinations of $\mu$ and $\sigma$ (foresight $=\mathbf{3 0})$

Fig. 11 plots the year in which T3 appears in the entire system with different combinations of $\mu$ and $\sigma$ when all agents have a foresight of 30 years. The left part of Fig. 11 shows the years in which $\mathrm{T} 3$ appears in cases in which there is no technological spillover effect, and the right part shows those in the presence of a technological spillover effect. As shown in Fig. 11, as $\mu$ increases, the year in which T3 appears in the entire system tends to be later. However, as $\sigma$ increases, whether the year in which T3 appears in the entire system is brought forward or postponed is very complicated. When there's no technological spillover effect in the system, how T3 diffuses in the entire system is actually the average of diffusions of different agents, and it highly depends on the random values assigned to a certain distribution. Thus, as $\sigma$ increases, the year in which T3 appears can be brought forward, or postponed, or mostly unchanged, as shown in the left part of Fig. 11. When there is a technological spillover effect, as $\sigma$ increases, the year T3 appears in the entire system becomes earlier. This is because as $\sigma$ increases, it allows more agents to be more risk-taking and adopt T3 earlier, other agents could benefit from their cumulative experience earlier, and in the entire system, T3 appears earlier.

On the contrary, comparing the left and right part of Fig. 11, when there's a technological spillover effect, the year in which T3 appears is brought forward in most cases. For example, when $\mu=1$ and $\sigma=1.5$, the year T3 appears is brought forward for about 10 years. However, when $\sigma=0.1$ and $\mu=1$ or $\mu=4$, the year T3 appears is postponed. This is because when $\sigma$ is comparatively smaller, most agents are very similar, and there is a possibility that the technological spillover effect will strength the lock-in of T2 and thus postpones the adoption of T3.

As shown in Figs. 6, 10, and 11, in the cases in which all of the agents have the same foresight, the existence of a technological spillover effect does not always bring forward the adoption of T3 in the entire system. 


\subsection{Technology adoption with heterogeneity in both foresights and risk attitudes}

After exploring how the adoption of T3 is influenced by heterogeneity in agents' foresights and risk attitudes respectively, we analyze the adoption of $\mathrm{T} 3$ with heterogeneity in both foresights and risk attitudes. In the simulations presented in this subsection, the agents are heterogeneous in terms of both foresights and risk attitudes: Each agent's foresight is given a random value from the set $\{20,30$, $40,50,60,70,80,90\}$, and each agent' risk attitude is a random value that follows a normal distribution. Each agent's objective function is denoted using Eq. (14), i.e., considering the uncertainty in technological learning. Again, when there is a technological spillover effect, the technological spillover rate $(\theta)$ is set to 0.5 .

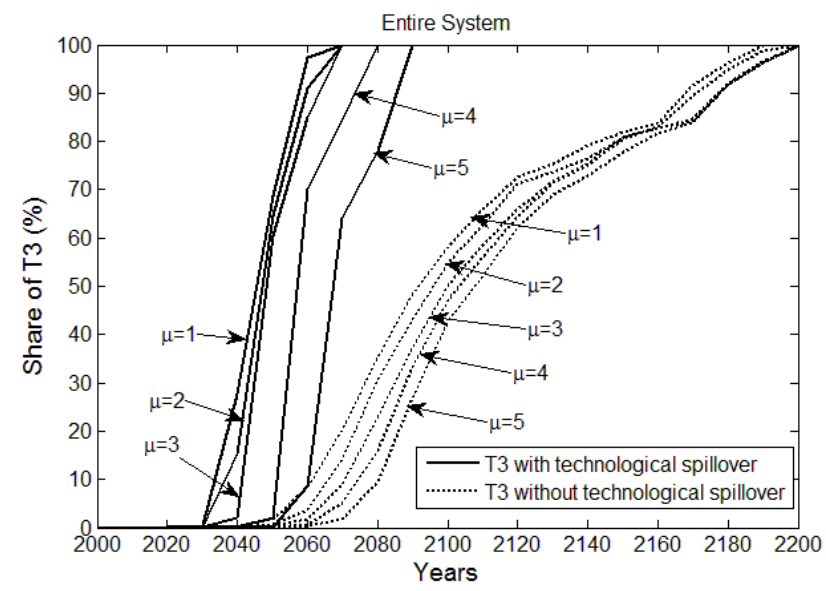

Figure 12. Adoption of $\mathrm{T} 3$ in the entire system with heterogeneity in agents' foresight and risk attitudes with different $\mu(\sigma=0.5)$

Fig. 12 shows how T3 diffuses in the entire system when agents are heterogeneous in terms of both foresights and risk attitudes with different $\mu$ (mean of the agents' risk attitude) and with the same $\sigma=0.5$. From Fig. 12 we can see that, as $\mu$ increases, the year T3 appears becomes later. In addition, when there is no technological spillover effect, although T3 starts to diffuse and completes the diffusion at almost the same time, a larger $\mu$ slightly lowers the diffusion level of T3 at each time point during the diffusion. Furthermore, it can be observed that the technological spillover effect significantly shortens the diffusion time of T3. For example, when the $\mu$ is 1 , it takes more than 100 years to complete the diffusion of T3 in the absence of a technological spillover effect, and it takes only approximately 40 years to complete the diffusion of T3 when there is a technological spillover effect.

Comparing Fig. 6, Fig. 7, Fig. 11, and Fig. 12, we can summarize that when agents are relative homogenous (the case in Fig.6 and the cases in Fig. 11 with very small $\sigma$ ), the technological 
spillover effect does not bring forward the adoption of T3 very much, and when agents are heterogeneous, the technological spillover effect accelerates the adoption of T3 quite a lot.

\subsection{Technology adoption with different technological spillover rates}

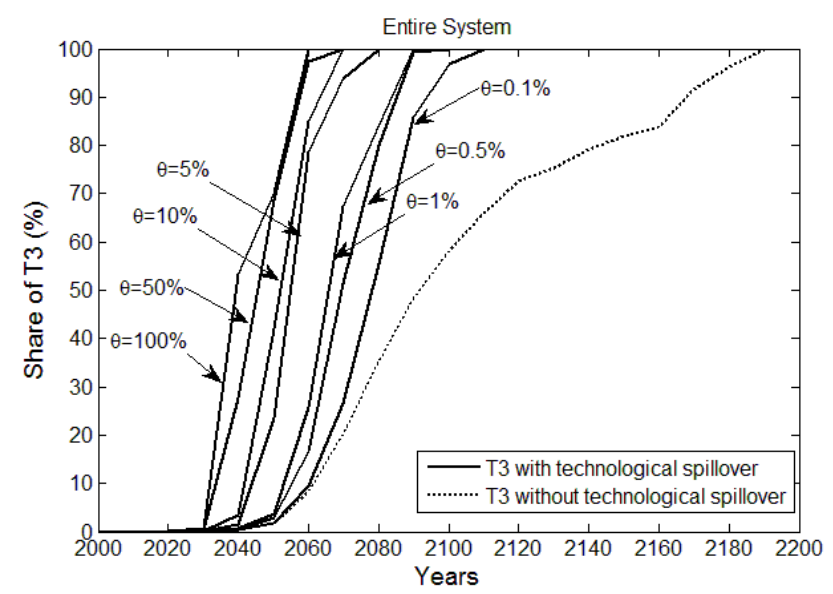

Figure 13. Adoption of T3 in the entire system with different $\theta$

Finally, we analyze the adoption of T3 with different technological spillover rates. Fig. 13 shows the diffusion of T3 in the entire system with different values of the technological spillover rate $\theta$. In the simulations presented in Fig. 13, each agent's foresight is a random value from the set $\{20,30,40$, $50,60,70,80,90\}$, each agent' risk attitude is a random value following a normal distribution with $\mu=1$ and $\sigma=0.5$, and each agent's objective function is denoted using Eq. (14), i.e., considering the uncertainties in technological learning. As shown in Fig. 13, compared with T3's diffusion in the absence of a technological spillover effect, even a very small technological spillover rate would significantly shorten the diffusion time of T3 in the entire system. A $0.1 \%$ technological spillover rate could shorten the diffusion time of T3 by 80 years, and as the value of $\theta$ increases from $0.1 \%$ to $100 \%$, the shortened diffusion time of T3 varies from 80 years to about 110 years.

\section{Conclusions}

The traditional operational optimization models of systematic technology adoption commonly assume the existence of a global social planner and ignore the existence of heterogeneous decision makers who interact with each other. This paper presents an optimization model of technology adoption with multiple agents that make decisions simultaneously. The agents are heterogeneous in terms of the length of their foresight and their attitudes toward the risk that results from uncertain technological learning, and there is technological spillover among them. This paper first illustrates that although a well recalibrated representative model can perform well enough when the interest is placed on aggregate variables, it could react to a policy (a carbon tax in this paper) differently from the multi-agent model. Using the multi-agent model, this paper explores how the agents' 
heterogeneities and the technological spillover effect influence the adoption of new advanced technology, and the main findings are the following:

- For optimization models of systematic technology adoption, the assumption of the existence of multiple decision makers implies a slower adoption of advanced technologies than the assumption of the existence of a global social planner.

- When the agents are the same, i.e., with the same foresight and the same risk attitude, the technological spillover effect does not always bring forward the adoption of advanced technology. On the contrary, in some cases, it will postpone the adoption of the advanced technology because it enhances the lock-in effect on previous technologies.

- When agents are heterogeneous, i.e., they have different foresight and different risk attitudes, even a small technological spillover rate can significantly accelerate the adoption of the advanced technology because, in the presence of heterogeneous foresight and risk attitudes, there are always some agents who adopt the advanced technology early, which benefits others in the presence of a technological spillover effect.

\section{Acknowledgements}

This research was sponsored by NSFC (No. 71125002 \& 71571069) and Fundamental Research Funds for the Central Universities (No. 222201522019).

\section{References}

[1] Arrow, K.J. (1962). The economic implications of learning by doing. The Review of Economic Studies, 29(3), 155-173.

[2] Arthur, W.B. (1989). Competing Technologies, Increasing Returns, and Lock-in by Historical Events. Economic Journal, 99 (394), 116-131.

[3] Azar, C., Lindgren, K., \& Andersson, B.A. (2003). Global energy scenarios meeting stringent CO2 constraints - cost-effective fuel choices in the transportation sector. Energy Policy, 31(10), 961-976.

[4] Bagozzi, R.P., Davis, F.D., \& Warshaw, P.R. (1992). Development and test of a theory of technological learning and usage. Human Relations, 45(7), 660-686.

[5] Barreto, L., \& Kypreos, S. (2002). Multi-regional technological learning in the energy systems MARKAL model. International Journal of Global Energy Issues, 17(3), 189-213.

[6] Bass, F.M. (1969). A new product growth model for consumer durables. Management Science, 15, 215-227.

[7] Chen, H., \& Ma, T. (2014). Technology adoption with limited foresight and uncertain technological learning. European Journal of Operational Research, 239(1), 266-275.

[8] Chi C., Ma T., Zhu B. (2012). Toward a low-carbon economy: Coping with technological bifurcations with a carbon tax. Energy Economics, 34(6), 2081-2088. 
[9] Davis, F.D. (1989). Perceived usefulness, perceived ease of use, and user acceptance of information technology. MIS Quarterly, 13(3), 319-340.

[10] Dodds P.E. (2014). Integrating housing stock and energy system models as a strategy to improve heat decarbonisation assessments. Applied Energy. 132:358-369.

[11] Freeman, C. (1994). The economics of technical change. Cambridge Journal of Economics, 18, $463-514$.

[12] Grübler, A., \& Gritsevskyi, A. (1998). A model of endogenous technological change through uncertain returns and learning (R\&D and investments). Working paper. IIASA, Laxenburg, Austria. < http://www.iiasa.ac.at/Research/TNT/WEB/endog. pdf $>$.

[13] Hedenus, F., Azar, C., \& Lindgren, K. (2006). Induced technological change in a limited foresight optimization model. Special Issue: Endogenous Technological Change and the Economics of Atmospheric Stabilisation, The Energy Journal, The Quarterly Journal of the IAEE's Energy Economics Education Foundation, 27, 109-22.

[14] Keppo, I., \& Strubegger, M. (2010). Short term decisions for long term problems-the effect of foresight on model based energy systems analysis. Energy, 35, 2033-2042.

[15] Krusell, P., \& Smith, A.A. (2010). Quantitative macroeconomic models with heterogeneous agents. Advances in Economics and Econometrics: Theory and Applications, Ninth World Congress, Volume I, pp. 298-340.

[16] Ma, T. (2010). Coping with uncertainties in technological learning. Management Science, 56(1), 192-201.

[17] Ma, T., \& Nakamori, Y. (2009). Modeling technological change in energy systems - From optimization to agent-based modeling. Energy, 34(7), 873-879.

[18] Manne, A. S., \& Barreto, L. (2004). Learn-by-doing and carbon dioxide abatement. Energy Economics, 26(4), 621-633.

[19] Martinsen, D., Krey, V., \& Markewitz, P. (2007). Implications of high energy prices for energy system and emissions - the response from an energy model for Germany. Energy Policy, 35(9), 4504-15.

[20] McDonald, A., \& Schrattenholzer, L. (2001). Learning rates for energy technologies. Energy Policy, 29(4), 255-261.

[21] Messner, S., Golodnikov, A., \& Gritsevskyi, A. (1996). A stochastic version of the dynamic linear programming model MESSAGE III. Energy, 21 (9), 775-784.

[22] Messner, S., \& Strubegger, M. (1994). The energy model MESSAGE III. In J. F. Hake, M. Kleemann, W. Kuckshinrichs, D. Martinsen, \& M. Walbeck (Eds.), Advances in systems analysis: Modelling energy-related emissions on a national and global scale (pp. 29-49). Jülich, Germany: Forschungszentrum Jülich.

[23] Metcalfe, S. (1987). Technical change. In: Eatwell, J., Milgate, M., Newman, P. (Eds.), The New 
Palgrave. In: A Dictionary of Economics, vol. 4, (pp. 617-620). Macmillan, London, UK.

[24] Mokyr, J. (1990). The Lever of Riches: Technological Creativity and Economic Progress. Oxford University Press, Oxford.

[25] Rogers, E.M. (1962). Diffusion of Innovations. Glencoe: Free Press.

[26] Seebregts, A.D. (2001). Energy/environmental modelling using the MARKAL family of models. Proc. OR2001 Conference, Energy, and Environment Session, Duisburg, Germany, September 3-5.

[27] Schwoon, M. (2008). Learning by doing, learning spillovers and the diffusion of fuel cell vehicles. Simulation Modelling Practice and Theory. 16:1463-1476.

[28] World Bank. (2000). Entering the 21st Century: world development report 1999/2000. World Bank, Washington, DC, USA. 